\title{
Differentially expressed microRNAs under imidacloprid exposure and identification in Sitobion avenae
}

Xi-Ling Chen ( $\square$ chenxiling456@126.com )

Henan Institute of Science and Technology

Baizhong Zhang

Henan Institute of Science and Technology

Junjie Liu

Henan Instiute of Science and Technology

Liuyang Lu

Henan Institute of Science and Technology

Lanfen Xie

Henan Institute of Science and Technology

Shouping Zhang

Henan Institute of Science and Technology

Research article

Keywords: MicroRNAs (miRNAs), Sitobion avenae, imidacloprid, resistance

Posted Date: May 27th, 2019

DOI: https://doi.org/10.21203/rs.2.9874/v1

License: (c) (i) This work is licensed under a Creative Commons Attribution 4.0 International License.

Read Full License 


\section{Abstract}

Backgroud: MicroRNAs (miRNAs), which are as short single-stranded non-coding RNAs, coudl regulate the expression of target genes, especially regulation or metabolism of endogenous or xenobiotic compounds. Results: The de novo assembly of the transcriptomes was obtained through Illumina short-read sequencing technology in Sitobion avenae. 57 miRNAs, of which 36 were known and 21 were novel were identified. Quantitative expression levels of miRNA showed that the expression of 5 miRNAs were significant up-regulation, and the expression of 11 miRNAs were significant down-regulation in the nymph

of S. avenae treated by imidacloprid in comparison with the control, respectively. The candidate transcript target genes in S. avenae that could be regulated by these miRNAs were also carried out. The functions of the miRNAs, which could potentially regulate the target genes participated in the metabolism, regulatory or detoxification of S. avenae were clarified based on Gene Ontology and KEGG pathway. The effects of the miRNAs identified api-miR-1000, api-miR-316, and api-miR-iab-4 on susceptibility of S. avenae to imidacloprid was determined. The abundance of api-miR-1000, api-miR-316, and api-miR-iab-4 modulated by the addition of their own inhibitors to the artificial diet could change significantly the susceptibility of S. avenae to imidacloprid, which further proved that the regulatory affect of these miRNAs on regulation or metabolism of insecticides. Conclusion: It suggested that differentially expressed microRNAs under the stress of imidacloprid could play critical regulatory role in the resistance of S. avenae to imidacloprid.

\section{Background}

MicroRNAs (miRNAs) are between 18 and 23 nucleotides in length and have been shown to be responsible for the post-transcriptional regulation of mRNAs. miRNA could silence expression of target genes by complementarily binding to $5^{\prime} / 3^{\prime}$ untranslated regions (UTRs) or coding sequences. ${ }^{1-5}$ UTRs of target mRNAs were binded mainly through imperfect complementary base pairing. ${ }^{6}$ The exact match is considered to be related to target specifcity. ${ }^{7}$

Studies have shown that miRNAs could regulate most of mammalian protein-coding gene ${ }^{8}$ which could be critical mediators in a series of physiological and developmental pathways, ${ }^{9-12}$ i.g apoptosis, cell proliferation, morphogenesis, and the metabolism of compounds. ${ }^{5,13}$

MiRNAs could mediate the detoxifcation/metabolism of xenobiotics through expression regulation of the detoxification genes, including nuclear receptors and xenobiotic-metabolizing enzymes, ${ }^{5}$ i.g human UDPglucuronosyltransferase (UGT) 1A was proved to be mediated post-transcriptionally by mir-491-3p to metabolize raloxifene metabolites ${ }_{,}{ }^{14}$ human P450 CYP1A1 related to the metabolism of carcinogenic and metabolites can be mediated post-transcriptionally by miR-892a. ${ }^{15}$

Previous reports conducted in Plutella xylostella indicated that miRNAs could be involved in chlorantraniliprole resistance by regulating the expression quantity of UGT genes. ${ }^{16}$ UGT2B7 and UGT2B15 genes, which were proved to be mediated post-transcriptionally by miRNA; ${ }^{17}$ Culex pipiens 
miRNAs are involved in the pyrethroid resistance by regulating the expression quantity of P450 genes. ${ }^{18}$, ${ }^{19}$ There are many miRNAs known involved in mediating the metabolic enzymes of xenobiotics in animals, while the affects of miRNAs on regulation of detoxifcation/metabolism of xenobiotics in pests is less understood.

The grain aphid, Sitobion avenae, is as the main pest of wheat distributed worldwide, which not only harms by sucking crop juice, but also spreads yellow dwarf virus disease, and leads to serious economic loss The control of this aphid has depended largely on the use of chemically synthesized pesticides. ${ }^{20}$ Imidacloprid is as one of neonicotinoids that targets sucking pests, such as aphids, green leaf bug and so on. ${ }^{21}$ It has been prevalently applied in wheat field to wheat aphid in China. So, S. avenae has

developed serious resistance to imidacloprid. ${ }^{22}$

Here, we performed high-throughput sequencing of short RNA libraries of control and imidacloprid treatment to detect differentially expressed microRNAs, and the functions of the miRNAs in regulating genes involved in the metabolism/ detoxification of $S$. avenae to xenobiotics. This will help understanding the roles of miRNAs in the regulation of the resistance to imidacloprid in insects.

\section{Methods}

\subsection{Culture condition}

The susceptive strain of $S$. avenae used was from the greenhouse for more 10 years, and has never been exposed to any insecticides. The culture condition was mentioned as previously. ${ }^{23}$

\subsection{Imidacloprid treatments}

Leaf-dipping with aphids method was conducted with minor modifications as previously described Chen et al. ${ }^{24}$ Leaf-dipping with $3^{\text {rd }}$ instar nymphs aphids were immersed in the imidacloprid solution $\left(0.5 \mathrm{mg} \mathrm{L}^{-}\right.$ $\left.{ }^{1}\right)$ for 5-10 s. And their imidacloprid-treated counterparts treated with distilled water as the control were also collected. Surviving aphids ( $\mathrm{N}=6$ for each sample), including imidacloprid challenge and counterparts were collected at the same time points $(24 \mathrm{~h})$.

\subsection{RNA isolation, miRNA library construction and Illumina sequencing}

Total RNA was extracted from controls and imidacloprid treatments of $S$. avenae using TRIzol reagent (Invitrogen, Shanghai, China) following the manufacturer's protocol and re-suspended in nuclease-free water $1 \%$ agarose gels were monitored to determine if RNA extracted was degradated and contaminated, and then purity, concentration and integrity of RNA were measured by NanoDrop 2000 spectrophotometer (Thermo Scientific, Wilmington, DE), Qubit ${ }^{\circledR}$ RNA Assay Kit in Qubit ${ }^{\circledR} 2.0$ Flurometer (Life Technologies, CA, USA) and RNA Nano 6000 Assay Kit of the Agilent Bioanalyzer 2100 system (Agilent Technologies, 
CA, USA), respectively. Approximately $3 \mu \mathrm{g}$ of total RNA as input material of each sample was used for constructing the small RNA library and conducted in Novogene, Beijing, China as previous description ${ }^{25}$.

\subsection{Bioinformatics analysis, miRNA prediction, and miRNA target prediction.}

S. avenae miRNAs, were confirmed by miRDeep2 software. ${ }^{26}$ Raw reads from the 4 libraries, including imidacloprid treatments and controls (2 biological replicates of each sample), were sent as insert into miRDeep2, and then the data (each library) was further separately analyzed. The default options and settings were used to performed the miRDeep2 analysis. And the sequences with polyA tails of the raw reads, the length of miRNAs ranging from 18 to $30 \mathrm{nt}$ was selected for further being analyzed after the adaptor sequences was trimmed, and removed snRNA, rRNA, snoRNA and tRNA. The sequences, mapping to the mature miRNAs in the premiRNAs and miRBase, and mapping to the Acyrthosiphon pisum genome would be identified to be as known mature miRNAs. The clean reads identified of $S$. avenae transcriptome has been sent to NCBI/SRA database, the accession number was SRP182781. Miranda performed the target genes prediction of miRNA. ${ }^{27}$ The putative mRNAs that targeted by miRNAs were predicted against $S$. avenae transcriptome nucleotide sequences. 2 software programs were conducted for miRNA target prediction, miRanda ${ }^{28}$ and RNAhybrid. ${ }^{29}$ The predicted miRNA target genes would be selected for further analysis. And then the target genes predicted would be aligned by the BLASTX program of NCBI, as well as mapping and annotation of the gene sequences were conducted using BLAST2GO. ${ }^{30}$ The Gene Ontology (GO) database, Genomes (KEGG) pathway and Kyoto Encyclopedia of Genes were used to further analyse putative identified target genes of miRNAs.

\subsection{Differential expressed miRNAs between controls and imidacloprid treatments of $S$. avenae}

In order to obtain the miRNAs of aphids affected by imidacloprid, the expression level of miRNAs was performed between controls and treatments of $S$. avenae. The edgeR software (3.10.2) obtained from Bioconductor version 3.1 was used to analyze the read counts of identified miRNA. ${ }^{31}$

The P-values was calculated by the Benjamini-Hochberg method. ${ }^{32}$ P-value corrected was set at 0.05 as the threshold for expressed difference by default significantly. Normalization of miRNA counts between libraries of controls and treatments was executed according to the total number of reads across libraries in silico. Normalized expression $=$ Actual miRNA count/Total count of clean reads $\times 10^{6}$. A false discovery rate (FDR) of the miRNAs $<0.05$, and a fold change $\geq 2$ was deemed to be significant.

\subsection{Quantitative real-time PCR (qPCR) validation}

To validate the miRNA data obtained by the deep sequencing, 3 miRNAs were selected to confirm their expression by qPCR. Total RNA, first strand cDNA synthesized, SYBR Green Master Mix (miScript SYBR Green PCR Kit, Qiagen) and qPCR procedure determined was described as previously. ${ }^{33}$ Relative expression quantification was calculated by the $2^{-\Delta \Delta C t}$ method. ${ }^{34} U 6$ snRNA was used to normalize expression quantiy of miRNAs as an endogenous control. The primers designed were showed in Table 1. 


\subsection{MiRNA inhibitor feeding in vitro, and subsequent impacts on imidacloprid susceptibility}

The rearing method and the artificial diet used were as previously described. ${ }^{35}$ with minor modifications. Both ends of transparent glass tube were open, sterilized and used as the feeding device (4 cm in length, $2.5 \mathrm{~cm}$ diameter). $25 \%$ sucrose as artificial diet was fit into 2 layers of parafilm sealed in the one end of the feeding device, and then healthy apterous adults were transfered to the device for rearing covered by the mesh to prevent them from escaping. The aphids were reared under the condition described previously. Each sample contained three replications.

To evaluate the inhibitory effect of miRNAs after the feeding of miRNA inhibitors, the artificial diet with miRNA inhibitors at a $2.5 \mathrm{mM} / \mathrm{L}$ final concentration, 50 healthy apterous aphids were placed to the artificial diet mixed with miRNA inhibitor, and the NC-inhibitor (a negative control) were used as the control; Following the feeding of $24 \mathrm{~h}$, the aphids survival were collected for RNA extraction, 3 replicates were carried out.

To evaluate the affects of miRNAs modulated on sensitivity of $S$. avenae to imidacloprid, 50 healthy apterous adults were placed to the artificial diet that contained imidacloprid $(0.1 \mathrm{mg} / \mathrm{L})$ with a $2.5 \mathrm{mM} / \mathrm{L}$ final concentration miRNA inhibitor, the NC-inhibitor (a negative control) were used for the control. 3 replicates were carried out, and mortality was caculated at $48 \mathrm{~h}$. The miRNA inhibitors used were provided by Sangon Biotech Co., Ltd (Shanghai, China).

\section{Results}

\subsection{Illumina sequencing data analysis}

There were 14, 841,584, and 13, 964, 533 raw reads, identified from RNA libraries from the imidacloprid treatment and control (Table 2). The length of S. avenae sRNAs was between $18 \mathrm{nt}$ and $35 \mathrm{nt}$, comprising $5.28 \%$ and $5.08 \%$ of the all reads with $22 \mathrm{nt}$ size from the imidacloprid treatment and control (Fig. $1 \mathrm{~A}$ ). Afer discarding low quality sequences and adapter, The unique reads, $6,992,451$ ( $47.11 \%$ of total) and $6,349,363(45.47 \%)$ remained from the control and imidacloprid treatments libraries, respectively (Table 2). The distribution in length of unique reads filtered indicated a distribution in the 2 libraries (Fig. 1B), where constituted $3.75 \%$ and $3.72 \%$ of the total reads at $22 \mathrm{nt}$, respectively, where at $23 \mathrm{nt}$ constituted $5.18 \%$ and $6.23 \%$ of the total reads, respectively. Where in the maximum at 32 nt constituted $8.59 \%$ of the all reads of imidacloprid libraries, at 34 nt constituted $9.76 \%$ of the all reads of controls, which probably showed piRNA-like sRNAs in S. avenae. A little bit sRNAs had over a thousand reads, however most had less than 10 copies (Fig. 1C). sRNAs in S. avenae showed a significant bias at $5^{\prime}(96.99 \%$, and $97.51 \%$, respectively) and $3^{\prime}$ ends (53.38\%, and $55.32 \%$, respectively) in both libraries (Fig. 2) for the nucleotide U. .

\subsection{Sequence and predication of miRNAs}

According to the distribution in length of the reads, of sRNA libraries miRNAs at 22 nt accounted for $43.86 \%$ of all species of miRNAs (Fig. 1D). The query of the reads of putative miRNAs in S. avenae to miRNAs from A. pisum genomes in miRBase, the results showed 36 sequences conserved were identified 
(Table S1). In addition, the query of the reads filtered in S. avenae against mature miRNAs, showing that 26 miRNA sequence families were identified from the miRBase database (Fig. 3A). It is predicted that the miR-10 family has the largest members $(n=415)$, next let-7 $(n=401)$, and miR-2 $(n=180)$. Insects were shared with these miRNAs, with the majority of miRNAs shared with the known A. pisum miRNAs, while a fewer extent miRNAs trended for other insect orthologs (Fig. 3B).

There were a total of 21 novel miRNAs potentially obtained, and named the prefx 'PC' (predicted candidate) based on the adopted nomenclature (Table S2). These specifc miRNAs could be mapped to $S$. avenae transcriptome sequences. It was predicted from Mfold that stem-loop hairpin secondary structures $\left(<18 \mathrm{kcal} / \mathrm{mole}^{36}\right)$ were consist of miRNAs precursor sequences, and some predicted structures in S. avenae were shown in Fig. S1.

Differentially expressed miRNAs between imidacloprid treatments and controls were also determined according to normalized differences of Illumina read counts, 57 S. avenae miRNAs, 16 of which (28.1\%) were differential expression, including 11 down-regulation and 5 up-regulation (Table 3).

The PCR amplified products of api-miR-1000, api-miR-316, and api-miR-iab-4 indicated the expected size between 60 and $100 \mathrm{bp}$ (a single band). Subsequent analysis of qPCR results confirmed that the expressed level of Api-miR-1000 was 5.5- fold higher in imidacloprid treatments compared to controls. While the expression of Api-miR-316, and Api-miR-iab-4 were 0.09-, and 0.12-fold lower in imidacloprid treatments compared to controls, respectively. (Fig. S2). Overall, qPCR results of miRNAs were indicated similar ranging trends as that of the RNA-seq analyses.

\subsection{Target prediction of miRNAs.}

The 16 differentially expressed miRNAs in S. avenae, the hypothetical targets within the UTRs of transcripts from S. avenae for the miRNAs were predicted using Target Scan software (Table S3). Possible functions predicted of these hypothetical target genes were annotated by GO enrichment, which could be involved in molecular functions, biological processes and cellular components, like development processes (Table S4). There were $58 \mathrm{GO}$ terms, which were identified for predicted miRNA target genes according to GO level 2 (Fig. 4A), like the genes participated in metabolic processes.

These genes including 71 metabolic pathways predicted by KEGG enrichment analysis might be targeted by miRNA regulation (Fig. 4B). The transcript targets were predicted to orthologs of related insects, which showed that differentially expressed miRNAs between the control and the imidacloprid treatment in $S$. avenae could bind and promote the post-transcriptional regulation, including different biological pathways like xenobiotic metabolism (Table S5).

To confirm the potential function of miRNAs in the resistance of $S$. avenae to imidacloprid, the predicted target genes for the miRNAs that could participate in xenobiotic metabolism were further focused. Interestingly, target genes for some miRNAs were found to play critical roles in insects response to xenobiotics, like cytochrome P450s, UDP-glucuronosyltransferase, glutathione S-transferase, etc. (Table 
4). Some miRNAs predicted have a lot of putative target genes, and many putative target genes were regulated by multiple miRNAs. (Table S3).

\subsection{Modulation of miRNAs consequently impacts the susceptibility of $S$. avenae to imidacloprid}

The expression levels of miRNAs, api-miR-1000, api-miR-316, and api-miR-iab-4, was significantly depressed in $S$. avenae aphids fed miRNA inhibitors compared to the quantity in $S$. avenae fed on NCinhibitor and DEPC-water as controls (Fig. 5A). Mortality decreased significantly $21.73 \%$ in S. avenae aphids fed api-miR-1000 inhibitor under imidacloprid exposure compared to the controls. While the mortality increased significantly by $26.75 \%$ and $21.86 \%$ in S. avenae aphids fed api-miR-316, and api-miRiab-4 inhibitors under imidacloprid exposure compared to the controls, respectively (Fig. 5B).

\section{Discussion}

It is predicted miRNAs can regulate a lot of protein-coding genes. ${ }^{37-39}$ And it is demonstrated that miRNAs are involved in regulation of various biological processes. In this study, miRNAs in S. avenae that might play regulatory roles in target genes responses to imidacloprid were identified and assessed. The identification and functional analysis of miRNAs differentially expressed in imidacloprid treated $S$. avenae were conducted, which may provide potentially understanding the regulation/detoxification of imidacloprid in insects. So far, the sequencing of small RNA has been as a widely trial way to obtaining miRNAs from a series of organisms based on sequencing technology of high throughput. Li et al. ${ }^{40}$ found out 345 miRNAs from the winged and wingless $S$. avenae. While we only identified 57 miRNAs in the control and imidacloprid treatments of $S$. avenae. However, our sequencing data could also greatly enlarge the range of the available information and provide critical basis for further studying miRNAs related to insecticide resistance in $S$. avenae.

Our analysis on expression of read counts indicated that 16 miRNAs differentially expressed were identified between the imidacloprid treatment and control of $S$. avenae, significantly indicating that imidacloprid could modulate expression of miRNAs, suggesting a probable regulatory role of these miRNAs in the detoxication/metabolism of insecticides in S. avenae. Several up/down-regulated miRNAs in the imidacloprid treatment of $S$. avenae could be involved in the regulation of the metabolism of imidacloprid. i.g api-miR-1000, api-miR-316, and api-miR-iab-4 were up/down-regulated in the imidacloprid treatment of $S$. avenae, which suggests that they could be involved in the regulation of the metabolism of imidacloprid. Drosophila melanogaster miR-1000 of activity-dependent expression could indicate a mechanism of allowing neuronal activity to fine-tune the strength in the excitatory synaptic transmission, and regulate the expressed level of vesicular glutamate transporter, and load the glutamate into synaptic vesicles, ${ }^{41}$ D. melanogaster dme-miR-1000 was located in the intron of musashi, ${ }^{42}$ encoding an RNA-binding protein to regulate gene translation by prior expression in the nervous system; ${ }^{43}$ Honeybee miR-1000 could be involved in musashi function of the nervous system, ${ }^{44}$ ame-miR-1000 was identified first, which was expressed highly selective manner of a insect brain. 1 cytochrome P450 and 2 glutathione S-transferase genes could be recognized by mse-miR-316; ${ }^{45}$ The accumulation endogenous 
Ubx protein could be attenuated as miR-iab-4-5p ectopic expression, and a classical homeotic mutant phenotype could also be induced (the transformation of halteres into wings), ${ }^{46}$ Drosophila dme-miR-iab-4 and dme-miR-iab-8 as miRNAs of bithorax-complex (BX-C) HOX bunches could participate in regulation of reproduction and neural patterning through suppressing HOX gene targets. ${ }^{47}$

In order to further exploring the role of miRNAs in the regulating metabolism of imidacloprid, the hypothetical target genes for miRNAs identified were predicted, and many of them annotated could participate in a lot of biological processes. At the same time, several miRNAs from different families target genes were proved to be involved in the xenobiotic detoxication/metabolism. ${ }^{48-51}$ We also found that several of the miRNAs targeting the genes involved in xenobiotic detoxication/metabolism were expressed differentially between the imidacloprid treatment and control of $S$. avenae by combining our analysis of differential expressed miRNAs with the prediction of the target genes.

The mortality of $S$. avenae exposed to imidacloprid significantly decreased after their feeding api-miR1000 inhibitor. While the mortality of $S$. avenae exposed to imidacloprid significantly increased after their feeding api-miR-316, and api-miR-iab-4 inhibitors, respectively. This showed that miRNAs differential expressied may participate in metabolism of imidacloprid by modulating gene expression related to xenobitic metabolism.

In conclusion, of 57 miRNAs obtained from $S$. avenae, the transcript levels of 16 miRNAs were ranged in $S$. avenae treated by imidacloprid. The prediction of targeting the genes related to xenobiotic metabolism showed that differentially expressed miRNAs could participate in the detoxication/metabolism of imidacloprid, and this represents a crucial resource of new small RNA genomics for further study in $S$. avenae.

\section{Abbreviations}

miRNAs: MicroRNAs; UGT: UDP-glucuronosyltransferase; qPCR: Quantitative real-time PCR; BLASTX: Similarity search of the NCBI protein database using a translated nucleotide query; GO: Gene ontology; KEGG: Kyoto Encyclopedia of Genes and Genomes; nr: NCBI non-redundant protein sequences database; nt: NCBI nucleotide collection; UTRs: untranslated regions;SRA: Sequence Read Archive

\section{Declarations}

\section{Acknowledgements}

This work is supported by the National Key Research and Development Program of China (2017YFD0201700), the Key Science and Technology Program of Henan (Agriculture) (182102110053). We thank Z.Y. Huang for reviewing and improving the manuscript.

\section{Funding}


Not applicable

\section{Availability of data and materials}

The transcriptome clean reads and computationally assembled sequences from this study were submitted to the NCBI/SRA database, under accession number: SRX374716.

\section{Author contributions statement}

S.Z and X.C conceived and designed the experiments, B.Z., and J.L. performed the experiments, B.Z., and S.Z. analyzed the data and wrote the manuscript text. J.L., and L.L. participated in the data analysis. All authors reviewed the manuscript.

\section{Competing interest}

The authors declare that they have no competing interests.

\section{Consent for publication}

Not applicable.

\section{Ethics approval and consent to participate}

Not applicable.

\section{Author details}

College of Resources and Environment, College of Animal Science and Veterinary Medicine, Henan Institute of Science and Technology, Xinxiang 453003, P.R.China

\section{Additional information}

Table S1. Summary of conserved miRNA.

Table S2.Summary of predicted miRNA.

Table S3. The predicted targets for miRNAs in Sitobion avenae.

Table S4. GO annoation for identified Sitobion avenae miRNAs.

Table S5. KEGG analysis for targets of differentially expressed Sitobion avenae miRNAs.

Fig. S1. Predicted stem-loop structures for three novel miRNA precursors.

Fig. S2. qPCR validation of miRNAs potentially involved in resistance of Sitobion avenae to imidacloprid. 


\section{References}

1. Asgari S, MicroRNA functions in insects. Insect Biochemistry and Molecular Biology 43: 388-397 (2013).

2. Pritchard $\mathrm{CC}$, Cheng $\mathrm{HH}$ and Tewari $\mathrm{M}$, MicroRNA profiling: approaches and considerations. Nature Reviews Genetics 13: 358-369.

3. Yokoi T and Nakajima M, MicroRNAs as mediators of drug toxicity. Annual Review of Pharmacology and Toxicology 53: 377-400 ( 2013).

4. Bartel DP, MicroRNAs: target recognition and regulatory functions. Cell 136:215-33 (2009).

5. Rigoutsos I, New tricks for animal microRNAS: targeting of amino acid coding regions at conserved and nonconserved sites. Cancer Research 69: 3245-8 (2009).

6. Stark A, Kheradpour P, Parts L, Brennecke J, Hodges E, Hannon GJ and Kellis M, Systematic discovery and characterization of fly microRNAs using 12 Drosophila genomes. Genome research17: 18651879 (2007).

7. Wang X. Composition of seed sequence is a major determinant of microRNA targeting patterns. Bioinformatics 15, 1377-1383 (2014).

8. Krol J, Loedige I and Filipowicz W, The widespread regulation of microRNA biogenesis, function and decay. Nature Reviews Genetics 11: 597-610 (2010).

9. Suh N, Baehner L, Moltzahn F, Melton C, Shenoy A, Chen J and Blelloch R, MicroRNA function is globally suppressed in mouse oocytes and early embryos. Current Biology20: 271-277 (2010).

10. Xu C, Lu Y, Pan Z, Chu W, Luo X, Lin H, Xiao J, Shan H, Wang Z and Yang B, The muscle-specific microRNAs miR-1 and miR-133 produce opposing effects on apoptosis by targeting HSP60, HSP70 and caspase-9 in cardiomyocytes. Journal of Cell Science120: 3045-3052 (2007).

11. Burnside J, Ouyang M, Anderson A, Bernberg E, Lu C, Meyers BC, Green PJ, Markis M, Isaacs G, Huang E and Morgan RW, Deep sequencing of chicken microRNAs. BMC Genomics 9: 185 (2008).

12. Legeai F, Rizk G, Walsh T, Edwards O, Gordon K, Lavenier D, Leterme N, Méreau A, Nicolas J, Tagu D and Jaubert-Possamai S, Bioinformatic prediction, deep sequencing of microRNAs and expression analysis during phenotypic plasticity in the pea aphid, Acyrthosiphon pisum. BMC Genomics 11: 281 (2010).

13. Lema $\mathrm{C}$ and Cunningham MJ, MicroRNAs and their implications in toxicological research. Toxicology letters198: 100-105 (2010).

14. Dluzen DF, Sun D, Salzberg AC, Jones N, Bushey RT, Robertson GP and Lazarus P, Regulation of UDPglucuronosyltransferase 1A1 expression and activity by microRNA 491-3p. Journal of Pharmacology and Experimental Therapeutics348: 465-477 (2014).

15. Choi YM, An S, Lee EM, Kim K, Choi SJ, Kim JS, Jang HH, An IS, Bae S, CYP1A1 is a target of miR892a-mediated post-transcriptional repression. International Journal of Oncology 41:331-336 (2012).

16. Zhu B, Xu M, Shi H, Gao X, Liang P, Genome-wide identification of IncRNAs associated with chlorantraniliprole resistance in diamondback moth Plutella xylostella (L.). BMC Genomics18: 380 
(2017).

17. Papageorgiou I and Court MH, Identification and validation of the microRNA response elements in the 3'-untranslated region of the UDP glucuronosyltransferase (UGT) 2B7 and 2B15 genes by a functional genomics approach. Biochemical Pharmacology 146: 199-213 (2017).

18. Hong S, Guo Q, Wang W, Hu S, Fang F, Lv Y, Yu J, Zou F, Lei Z, Ma K, Ma L, Zhou D, Sun Y, Zhang D, Shen B, Zhu C, Identification of differentially expressed microRNAs in Culex pipiens and their potential roles in pyrethroid resistance. Insect Biochemistry and Molecular Biology 55:39-50 (2014).

19. Lei Z, Lv Y, Wang W, Guo Q, Zou F, Hu S, Fang F, Tian M, Liu B, Liu X, Ma K Ma L, Zhou D, Zhang D, Sun Y, Shen B, Zhu C, MiR-278-3p regulates pyrethroid resistance in Culex pipiens pallens. Parasitology Research 114: 699-706 (2015).

20. Chen M, Han Z, Qiao X and Qu M, Resistance mechanisms and associated mutations in acetylcholinesterase genes in Sitobion avenae (Fabricius). Pesticide Biochemistry and Physiology 87:189-195 (2007).

21. Wang K, Zhang M, Huang Y, Yang Z, Su S and Chen M, Characterisation of imidacloprid resistance in the bird cherry-oat aphid, Rhopalosiphum padi, a serious pest on wheat crops. Pest Management Science 74:1457-1465 (2018).

22. Zhang S, Shao ZY and Li YP, Monitoring of agricultural pest resistance and suggestions for scientific medicine in 2013. China Plant Protection 34:22-25 (2014).

23. Lu Y and Gao X, Multiple mechanisms responsible for differential susceptibilities of Sitobion avenae (Fabricius) and Rhopalosiphum padi (Linnaeus) to pirimicarb. Bulletin of Entomological Research 99: 611-617 (2009).

24. Chen X, Xia X, Wang H, Qiao K and Wang K, Cross-resistance to clothianidin and acetamiprid in the imidacloprid resistant strain of Aphis gossypii (Hemiptera: Aphididae) and the related enzyme mechanisms. Acta Entomologica Sinica 56: 1143-1151 (2013).

25. Xu LN, Ling YH, Wang YQ, Wang ZY, Hu BJ, Zhou ZY, Hu F, He KL, Identification of differentially expressed microRNAs between Bacillus thuringiensis Cry1 Ab-resistant and-susceptible strains of Ostrinia furnacalis. Scientific reports5: 15461(2015).

26. Friedlander MR, Mackowiak SD, Li N, Chen W and Rajewsky N, MiRDeep2 accurately identifes known and hundreds of novel microRNA genes in seven animal clades. Nucleic Acids Research 40:37-52 (2012).

27. Enright AJ, John B, Gaul U, Tuschl T, Sander C and Marks DS, MicroRNA targets in Drosophila. Genome Biology5: R1 (2003).

28. Betel D, Wilson M, Gabow A, Marks DS, Sander C, The microRNA. org resource: targets and expression. Nucleic acids Research36: D149-D153 (2008).

29. Rehmsmeier M, Steffen $P$, Höchsmann M, Giegerich R, Fast and effective prediction of microRNA/target duplexes. RNA10: 1507-1517 (2004).

30. Conesa A, Götz S, García-Gómez JM, Terol J, Talón M, Robles M, Blast2GO: a universal tool for annotation, visualization and analysis in functional genomics research. Bioinformatics 21: 3674- 
3676 (2005).

31. Robinson MD, McCarthy DJ, Smyth GK, edgeR: a Bioconductor package for differential expression analysis of digital gene expression data. Bioinformatics26: 139-140 (2010).।

32. Benjamini $Y$, Hochberg $Y$, Controlling the false discovery rate: a practical and powerful approach to multiple testing. Journal of the Royal statistical society: series B (Methodological)57: 289-300 (1995).

33. Zhang, B. Z., Ma, K. S., Liu, J. J., Lu, L. Y., Chen, X. L., Zhang, S. P., \& Gao, X. W. (2018). Differential expression of genes in greenbug (Schizaphis graminum Rondani) treated by imidacloprid and RNA interference. Pest Management Science. https://doi.org/10.1002/ps.5293

34. Pfaffl MW, A new mathematical model for relative quantification in real-time RT-PCR. Nucleic Acids Research29: e45-e45 (2001).

35. Gong YH, Yu XR, Shang QL, Shi XY and Gao XW, 2014. Oral delivery mediated RNA interference of a carboxylesterase gene results in reduced resistance to organophosphorus insecticides in the cotton aphid, aphis gossypii Glover. PloS One 9: e102823.

36. Zhang X, Zheng Y, Jagadeeswaran G, Ren R, Sunkar R and Jiang H, Identification and developmental profiling of conserved and novel microRNAs in Manduca sexta. Insect Biochemistry and Molecular Biology42: 381-395 (2012).

37. Cai ZG, Zhang SM, Zhang H, Zhou YY, Wu HB and Xu XP, Aberrant expression of microRNAs involved in epithelial-mesenchymal transition of HT-29 cell line. Cell biology internationa/ 37: 669-674 ((2013)

38. Liu Q, Tuo W, Gao H and Zhu XQ, MicroRNAs of parasites: current status and future perspectives. Parasitology Research 107: 501-507 (2010).

39. Truini A, Coco S, Alama A, Genova C, Sini C, Dal Bello MG,Barletta G, Rijavec E, Burrafato G, Boccardo $\mathrm{F}$ and Grossi F, Role of microRNAs in Malignant mesothelioma. Cellular and Molecular Life Sciences71: 2865-2878 (2014).

40. Li X, Zhang F, Coates B, Zhang Y, Zhou X and Cheng D, Comparative profiling of microRNAs in the winged and wingless English grain aphid, Sitobion avenae (F.)(Homoptera: Aphididae). Scientific reports 6: 35668 (2016).

41. Verma P, Augustine GJ, Ammar MR, Tashiro A and Cohen SM, A neuroprotective role for microRNA miR-1000 mediated by limiting glutamate excitotoxicity. Nature Neuroscience 18: 379-385 (2015).

42. Stark A, Kheradpour P, Parts L, Brennecke J, Hodges E, Hannon GJ, Kellis M, Systematic discovery and characterization of fly microRNAs using 12 Drosophila genomes, Genome Research 17:18651879 (2007).

43. Okabe M, Imai T, Kurusu M, Hiromi $Y$ and Okano $\mathrm{H}$, Translational repression determines a neuronal potential in Drosophila asymmetric cell division. Nature 411: 94-98 (2001).

44. Hori S, Kaneko K, Saito TH, Takeuchi H and Kubo T, Expression of two microRNAs, ame-mir-276 and1000, in the adult honeybee (Apis mellifera) brain. Apidologie42: 89-102 (2011). 
45. Zhang X, Zheng Y, Cao X, Ren R, Yu X and Jiang H, Identification and profiling of Manduca sexta microRNAs and their possible roles in regulating specific transcripts in fat body, hemocytes, and midgut. Insect Biochemistry and Molecular Biology 62: 11-22 (2015).

46. Ronshaugen M, Biemar F, Piel J, Levine M and Lai EC, The Drosophila microRNA iab-4 causes a dominant homeotic transformation of halteres to wings. Genes \& Development19: 2947-2952 (2005).

47. Garaulet DL, Castellanos MC, Bejarano F, Sanfilippo P, Tyler DM, Allan DW, Sánchez-Herrero E and Lai EC, Homeotic function of Drosophila Bithorax-complex miRNAs mediates fertility by restricting multiple Hox genes and TALE cofactors in the CNS. Developmental cel/29: 635-648 (2014).

48. Omiecinski CJ, Vanden Heuvel JP, Perdew GH and Peters JM, Xenobiotic metabolism, disposition, and regulation by receptors: from biochemical phenomenon to predictors of major toxicities. Toxicological Sciences 120: 49-75 (2011).

49. Li X, Schuler MA and Berenbaum MR, Molecular mechanisms of metabolic resistance to synthetic and natural xenobiotics. Annual Review of Entomology 52: 231-53 (2007).

50. Kaplanoglu E, Chapman P, Scott IM and Donly C, Overexpression of a cytochrome P450 and a UDPglycosyltransferase is associated with imidacloprid resistance in the Colorado potato beetle, Leptinotarsa decemlineata. Scientific Reports7: 1762. (2017)..

51. Tian F, Wang Z, Li C, Liu J and Zeng, X, UDP-Glycosyltransferases are involved in imidacloprid resistance in the Asian citrus psyllid, Diaphorina citri (Hemiptera: Lividae). Pesticide Biochemistry and Physiology 154: 23-31 (2019).

\section{Tables}

Table 1. Primers were used in the study

\begin{tabular}{|c|c|c|c|}
\hline Primer name & Sequence (5'-3') & Size/bp & Application \\
\hline \multirow[t]{2}{*}{ api-mir-1000 } & F:AGAGCACGATATTGTCCTGTCACA & \multirow[t]{2}{*}{73} & qPCR \\
\hline & R:TATGGTTGTTCACGACTGGTTCAC & & qPCR \\
\hline \multirow[t]{2}{*}{ api-mir-316 } & F:TTTGGTGTCTTTTTCCGCTTTG & \multirow[t]{2}{*}{69} & qPCR \\
\hline & R:GTGCAGGGTCCGAGGT & & qPCR \\
\hline \multirow[t]{2}{*}{ api-mir-iab-4 } & F:AGGCGAAGCGACGTATACTAAATG & \multirow[t]{2}{*}{68} & qPCR \\
\hline & R:GTGCAGGGTCCGAGGT & & qPCR \\
\hline \multirow[t]{2}{*}{ U6 } & F:CAGCACATATACTAAAATTGGAACG & \multirow[t]{2}{*}{76} & qPCR \\
\hline & R:ACGAATTTGCGTGTCATCC & & qPCR \\
\hline MircoRNA inhibitor N.C & CAGUACUUUUGUGUAGUACAA & 21 & miRNA function \\
\hline api-mir-1000 inhibitor & UACUGCUGUGACAGGACAAUAU & 22 & miRNA function \\
\hline api-mir-316 inhibitor & CGGCAGCAAAGCGGAAAAAGACA & 23 & miRNA function \\
\hline api-mir-iab-4 inhibitor & UCAGGAUACAUUUAGUAUACGU & 22 & miRNA function \\
\hline
\end{tabular}

Table 2. Distribution of miRNA reads from control and imidacloprid treatment of Sitobion avenae 


\begin{tabular}{ccc}
\hline Parameter & Controls & Imidacloprid treatments \\
\hline Raw reads & $14,841,584$ & $13,964,533$ \\
Clean reads & $14,224,837$ & $13,492,898$ \\
3ADT and length filter & $10,959,929$ & $9,305,973$ \\
rRNA & 253,777 & 247,747 \\
tRNA & 16 & 23 \\
snoRNA & 9,443 & 7,823 \\
snRNA & 25,993 & 24,246 \\
Mappable reads & $6,992,451$ & $6,349,363$ \\
\hline
\end{tabular}

Note: 3ADT: reads that were removed as 3ADT was not found and length was less than 15nt and was more than 35nt. Mappable reads: reads from the raw reads that were passed by many the digital filters .

Table 3. The differentially expressed miRNAs in small RNA libraries of Sitobion avenae 


\begin{tabular}{|c|c|c|c|c|c|}
\hline miR_Name & $\begin{array}{l}\text { Imidacloprid } \\
\text { treatment_FPKM }\end{array}$ & Control_FPKM & P-value & $\begin{array}{l}\log _{2}(\text { Fold } \\
\text { Change })\end{array}$ & Difference \\
\hline api-miR-998 & 4021.337154 & 14561.84926 & 0 & -1.8564 & Down \\
\hline Novel-28 & 435.4314115 & 2094.17899 & $\begin{array}{l}1.93 E- \\
286\end{array}$ & -2.2659 & Down \\
\hline api-miR-306 & 691.567536 & 2386.390012 & $\begin{array}{l}1.54 \mathrm{E}- \\
246\end{array}$ & -1.7869 & Down \\
\hline Novel-22 & 742.7947608 & 1801.967968 & $\begin{array}{l}1.83 E- \\
120\end{array}$ & -1.2785 & Down \\
\hline Novel-9 & 717.1811484 & 1704.564294 & $\begin{array}{l}1.79 \mathrm{E}- \\
110\end{array}$ & -1.249 & Down \\
\hline $\begin{array}{l}\text { api-miR- } \\
3047\end{array}$ & 332.9769618 & 779.2293917 & $\begin{array}{l}3.01 \mathrm{E}- \\
50\end{array}$ & -1.2266 & Down \\
\hline Novel-25 & 256.1361244 & 657.4747992 & $\begin{array}{l}6.09 \mathrm{E}- \\
49\end{array}$ & -1.36 & Down \\
\hline $\begin{array}{l}\text { api-miR- } \\
1000\end{array}$ & 128.0680622 & 0 & $\begin{array}{l}8.16 \mathrm{E}- \\
28\end{array}$ & 8.0008 & Up \\
\hline api-miR-316 & 0 & 48.70183698 & $\begin{array}{l}2.37 \mathrm{E}- \\
13\end{array}$ & -6.6059 & Down \\
\hline Novel-39 & 51.22722489 & 0 & $\begin{array}{l}4.65 E- \\
13\end{array}$ & 6.6788 & $\mathrm{Up}$ \\
\hline Nove-35 & 153.6816747 & 48.70183698 & $\begin{array}{l}2.31 \mathrm{E}- \\
12\end{array}$ & 1.6579 & Up \\
\hline $\begin{array}{l}\text { api-miR- } \\
\text { 263b }\end{array}$ & 0 & 24.35091849 & $\begin{array}{l}1.57 \mathrm{E}- \\
07\end{array}$ & -5.6059 & Down \\
\hline $\begin{array}{l}\text { api-miR- } \\
2796\end{array}$ & 0 & 24.35091849 & $\begin{array}{l}1.57 \mathrm{E}- \\
07\end{array}$ & -5.6059 & Down \\
\hline $\begin{array}{l}\text { api-miR-iab- } \\
4\end{array}$ & 0 & 24.35091849 & $\begin{array}{l}1.57 \mathrm{E}- \\
07\end{array}$ & -5.6059 & Down \\
\hline $\begin{array}{l}\text { api-miR- } \\
3015 a\end{array}$ & 25.61361244 & 0 & $\begin{array}{l}2.52 \mathrm{E}- \\
07\end{array}$ & 5.6788 & Up \\
\hline $\begin{array}{l}\text { api-miR- } \\
3042\end{array}$ & 76.84083733 & 24.35091849 & $\begin{array}{l}7.05 E- \\
07\end{array}$ & 1.6579 & Up \\
\hline
\end{tabular}

Table 4 MiRNAs target for putative genes related to xenobiotic metabolism in Sitobion avenae 


\begin{tabular}{|c|c|}
\hline miR_Name & Target genes related to xenobiotic metabolism \\
\hline $\begin{array}{l}\text { api-miR- } \\
998\end{array}$ & CYP3A19, glutathione S-transferase omega-1, glutathione S-transferase, C-terminal domain \\
\hline novel-28 & $\begin{array}{l}\text { Microsomal epoxide hydrolase, glutathione S-transferase, C-terminal domain, P450 (GC vitamin } \\
\text { D-binding protein), CYP4C1, CYP4V, CYP9Z4 }\end{array}$ \\
\hline $\begin{array}{l}\text { api-miR- } \\
306\end{array}$ & $\begin{array}{l}\text { alcohol dehydrogenase 4, UDP-glucuronosyltransferase 2B10, UDP-glucuronosyltransferase 2B7, } \\
\text { Cytochrome P450 (piezo-type mechanosensitive ion channel component 2), CYP4C1, Glutathione } \\
\text { S-transferase (EEF1G elongation factor 1-gamma), CYP6A14 }\end{array}$ \\
\hline novel-22 & $\begin{array}{l}\text { UDP-glucuronosyltransferase 2C1, UDP-glucuronosyltransferase 1-7C UDP- } \\
\text { glucuronosyltransferase 2B17, Glutathione S-transferase Mu 1, Glutathione S-transferase } \\
\text { (phosphatidylinositol-binding clathrin assembly protein LAP), CYP4g15, CYP4C1 }\end{array}$ \\
\hline novel-9 & $\begin{array}{l}\text { UDP-glucuronosyltransferase 2C1, carbonyl reductase 1, Microsomal glutathione S-transferase 1, } \\
\text { UDP-glucuronosyltransferase 1-7 }\end{array}$ \\
\hline $\begin{array}{l}\text { api-miR- } \\
3047\end{array}$ & $\begin{array}{l}\text { UDP-glucuronosyltransferase 2C1, UDP-glucuronosyltransferase 1-7, CYP315A1, CYP2U1, P450 } \\
\text { (piezo-type mechanosensitive ion channel component 2) }\end{array}$ \\
\hline novel-25 & $\begin{array}{l}\text { alcohol dehydrogenase class-3, S-(hydroxymethyl)glutathione dehydrogenase / alcohol } \\
\text { dehydrogenase, glutathione S-transferase, C-terminal domain, CYP3A19, }\end{array}$ \\
\hline $\begin{array}{l}\text { api-miR- } \\
1000\end{array}$ & $\begin{array}{l}\text { UDP-glucuronosyltransferase 2B7, UDP-glucuronosyltransferase 2B10, UDP-glucuronosyl } \\
\text { transferase 351A3, Glutathione S-transferase (phosphatidylinositol-binding clathrin assembly } \\
\text { protein LAP), Glutathione S-transferase (failed axon connections), Cytochrome P450 (piezo-type } \\
\text { mechanosensitive ion channel component 2), CYP4C1, CYP6A2, CYP6A14 }\end{array}$ \\
\hline $\begin{array}{l}\text { api-miR- } \\
316\end{array}$ & $\begin{array}{l}\text { UDP-glucuronosyltransferase 2B1, Cytochrome P450 (piezo-type mechanosensitive ion channel } \\
\text { component 2), CYP4C1, CYP305a1, Cytochrome bd terminal oxidase }\end{array}$ \\
\hline novel-39 & Glutathione S-transferase GSTZ1 \\
\hline novel-35 & $\begin{array}{l}\text { Glutathione S-transferase (phosphatidylinositol-binding clathrin assembly protein LAP), } \\
\text { Cytochrome P450 (piezo-type mechanosensitive ion channel component 2), CYP49A1, CYP6A14, } \\
\text { CYP315A1, CYP4C1, CYP6A13, CYP6J1 }\end{array}$ \\
\hline api-miR- & UDP-glucuronosyltransferase 2C1, UDP-glucuronosyltransferase 2A1, glutathione S-transferase \\
\hline $263 \mathrm{~b}$ & C-terminal domain, CYP6A13, CYP4C1, CYP4V2, CYP49A1, \\
\hline $\begin{array}{l}\text { api-miR- } \\
2796\end{array}$ & UDP-glucuronosyltransferase 2B18 \\
\hline $\begin{array}{l}\text { api-miR- } \\
\text { iab-4 }\end{array}$ & CYP314A1, CYP4C1, CYP6A13, CYP3A19 \\
\hline $\begin{array}{l}\text { api-miR- } \\
3015 a\end{array}$ & $\begin{array}{l}\text { alcohol dehydrogenase class-3, UDP-glucuronosyltransferase 2A1dehydrogenase, glutathione S- } \\
\text { transferase C-terminal domain, Glutathione S-transferase (phosphatidylinositol-binding clathrin } \\
\text { assembly protein LAP), Cytochrome P450 (piezo-type mechanosensitive ion channel component } \\
\text { 2), CYP3A19, }\end{array}$ \\
\hline $\begin{array}{l}\text { api-miR- } \\
3042\end{array}$ & Glutathione S-transferase (EEF1G elongation factor 1-gamma) \\
\hline
\end{tabular}

Page 16/22 
Figures
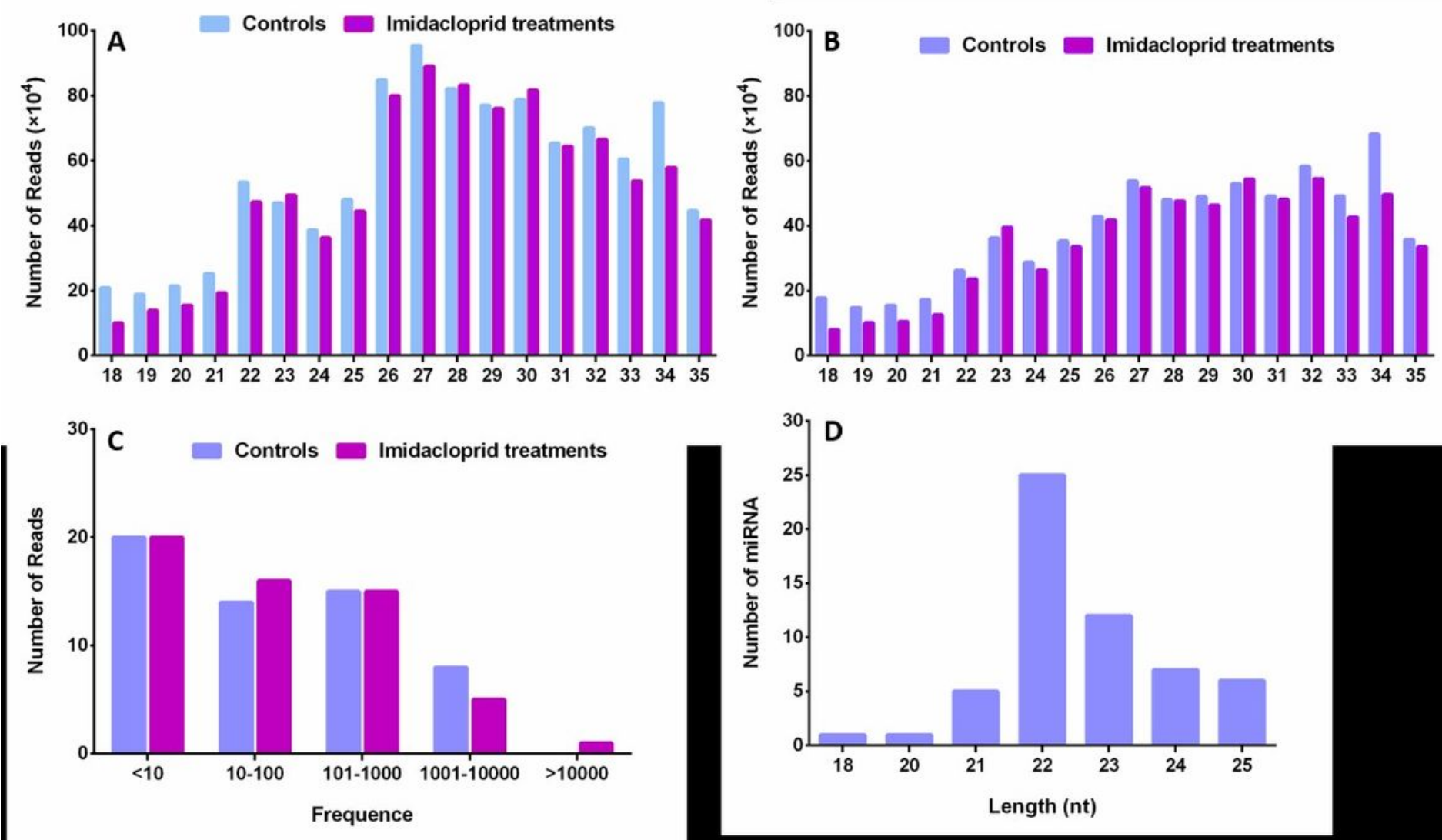

Figure 1

Characterization of miRNA sequences from Sitobion avenae. 

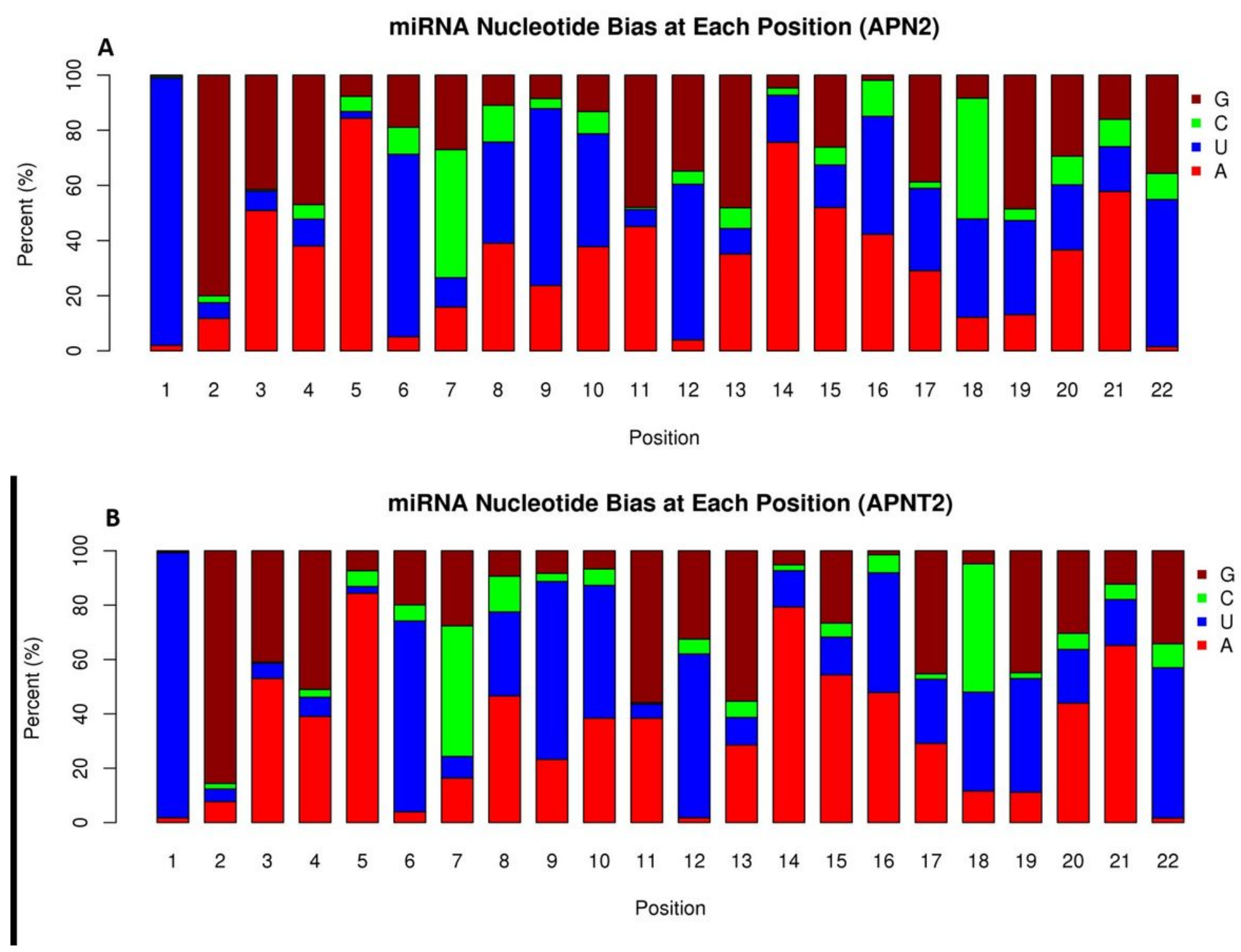

Figure 2

Nucleotide bias of predicated miRNAs from Sitobion avenae. 

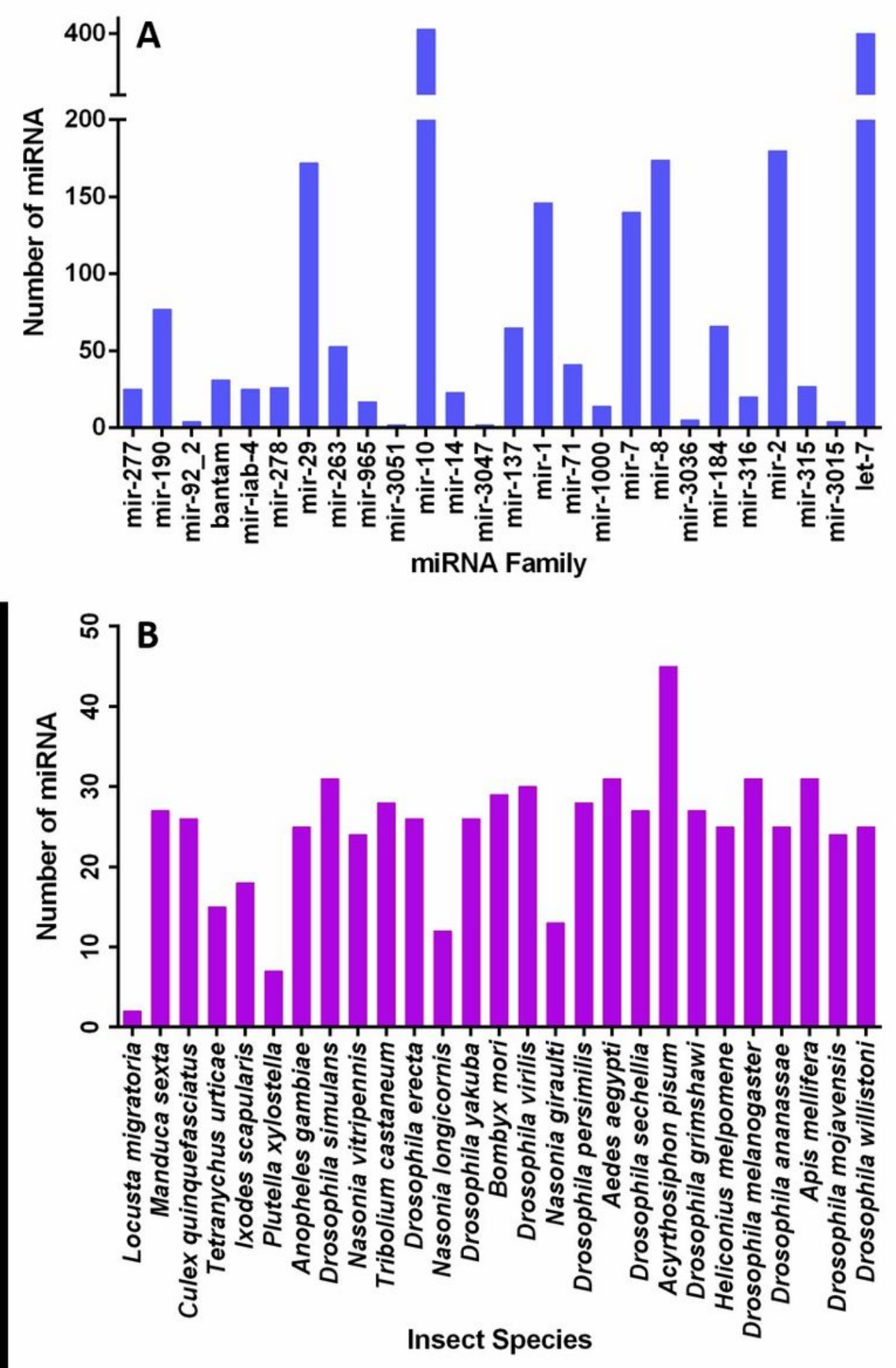

Figure 3

MiRNAs characterization of Sitobion avenae. 


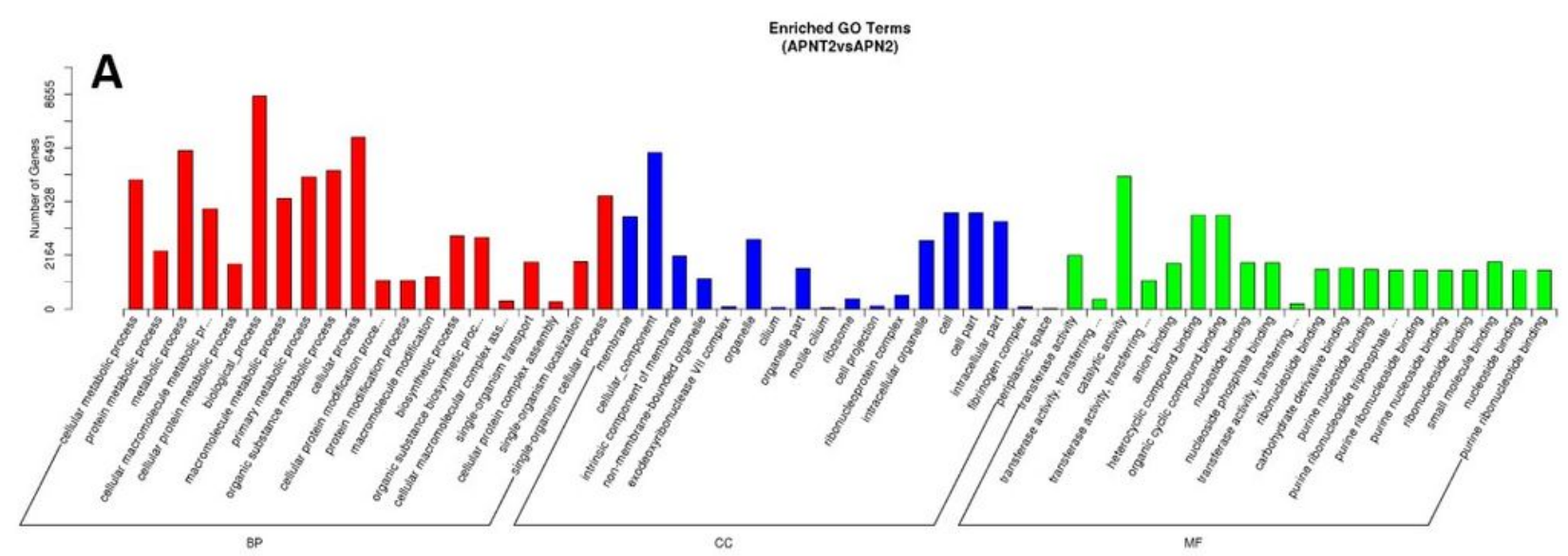

B

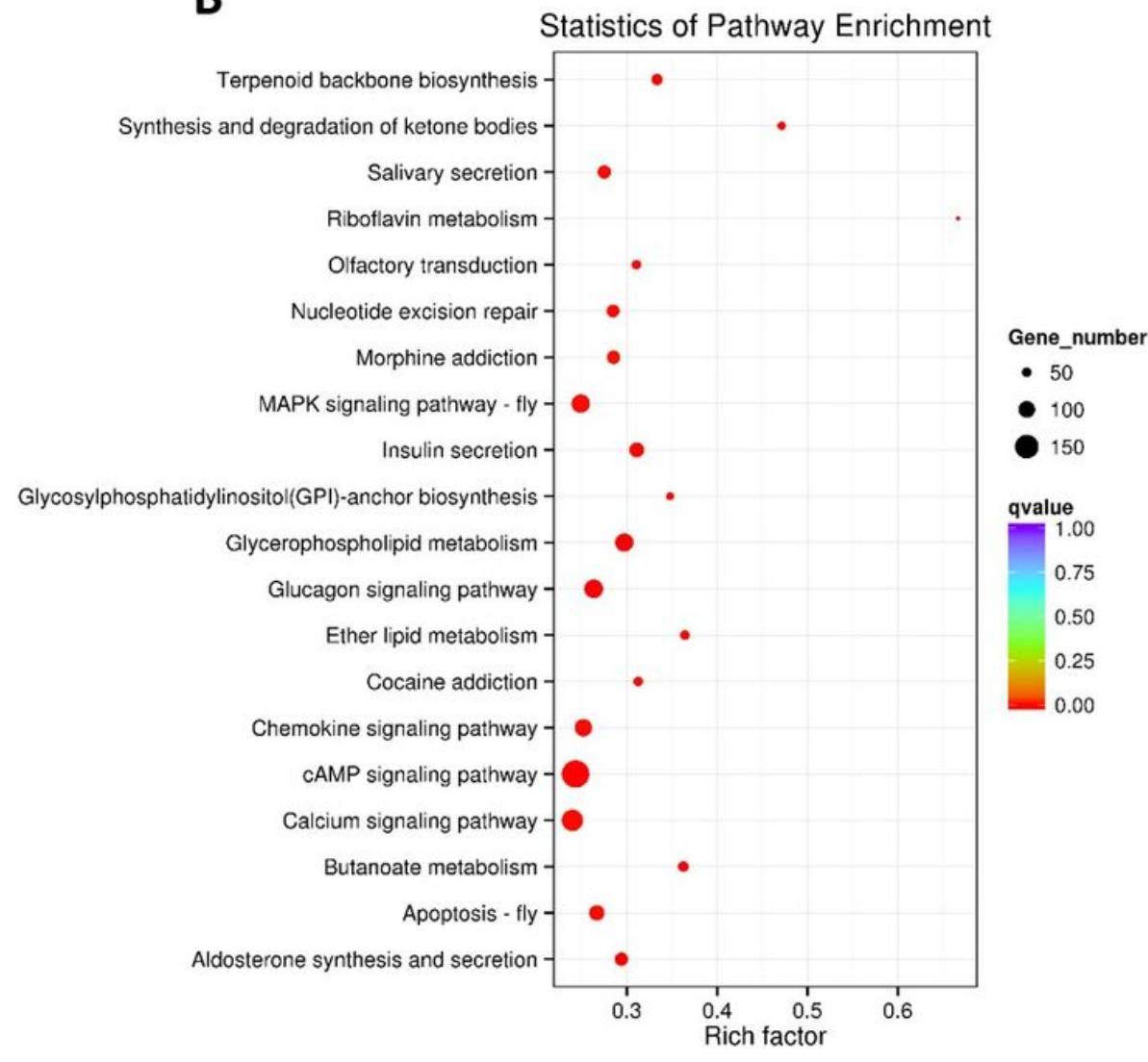

\section{Figure 4}

Histogram presentation of GO annoation and KEGG pathway for miRNAs identified from Sitobion avenae. 


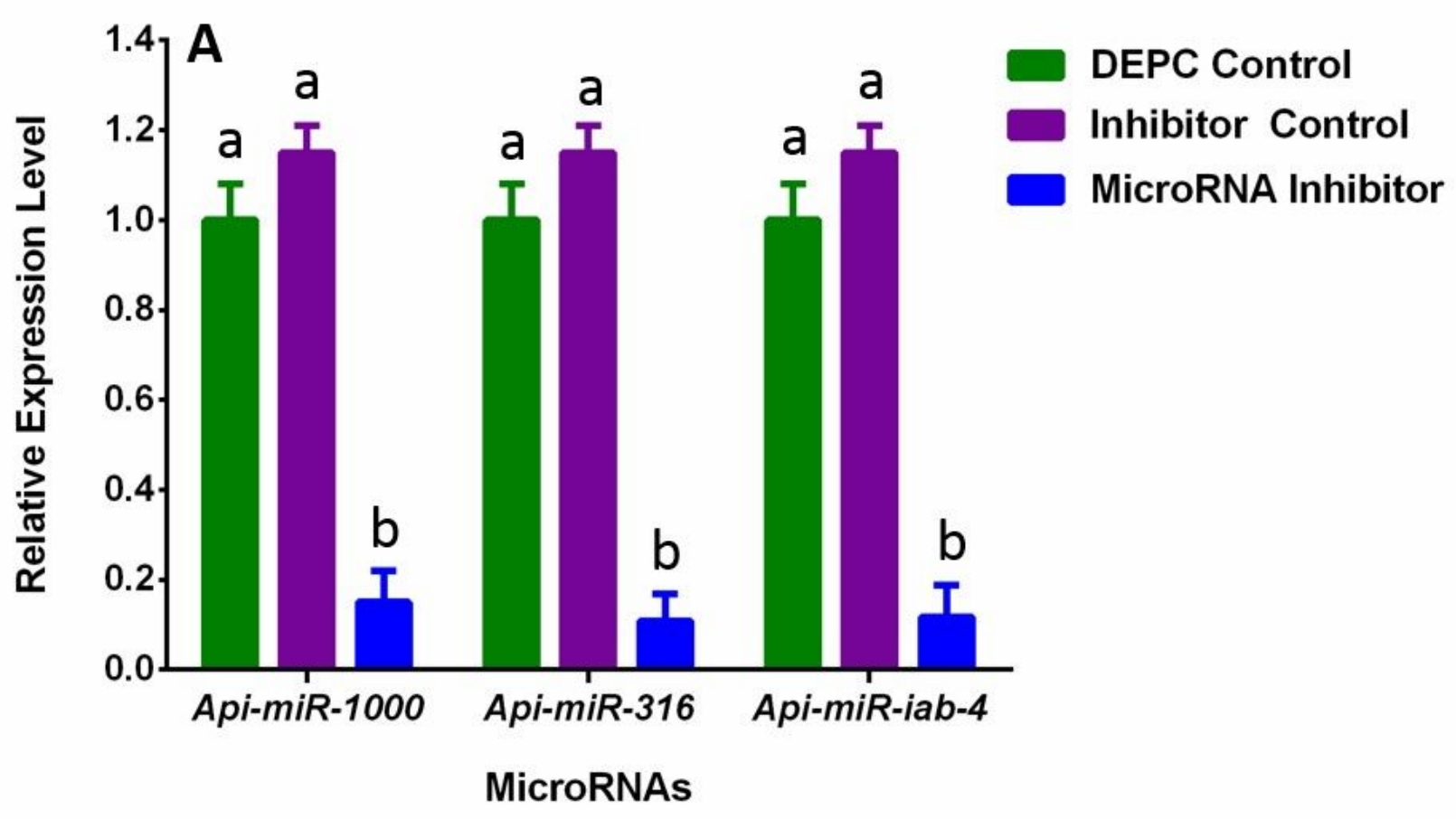

7

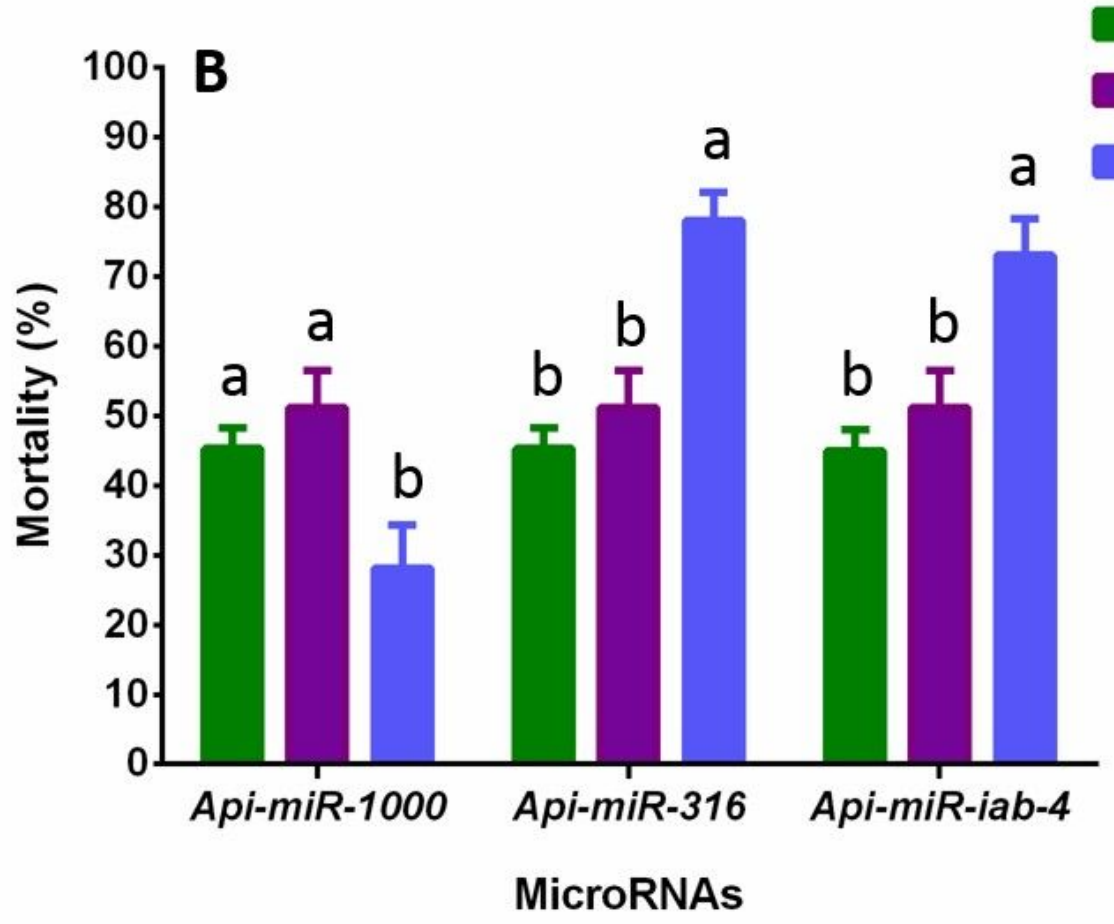

Imidacloprid+DEPC Control Imidacloprid+Inhibitor Control Imidacloprid+MicroRNA Inhibitor

Figure 5

Effects of miRNA modulation on susceptibility of Sitobion avenae to imidacloprid.

\section{Supplementary Files}

This is a list of supplementary files associated with this preprint. Click to download. 
- supplement1.docx

- supplement2.xIsx 